\title{
Synthesis, Antimicrobial and Antioxidant Evaluation of Novel 5, 6-dihydro- 3-(substituted phenyl) [1,3,4]thiadiazine-7-one Derivatives
}

\author{
Prabhakar Kumar Verma* \\ Department of Pharmaceutical Sciences, Maharshi Dayanand University, Rohtak, Haryana, India
}

\begin{abstract}
A series of 1, 2, 4-triazole derivatives (1-17, Scheme 1) was synthesized and evaluated for their antimicrobial and antioxidant potential. Results of antimicrobial screening of the synthesized compounds indicated that most of the synthesized compounds showed good activity as compare to the norfloxacin as antibacterial and fluconazole as antifungal standard drugs. Compounds 7 and 12 were found to be most effective against various strains used for the antimicrobial activity like S. aureus, B. subtilis, E. coli, Candida albicans and A. niger using tube dilution method having MIC $3.12 \mu \mathrm{g} / \mathrm{ml}$ for $S$. aureus and $06.25 \mu \mathrm{g} / \mathrm{ml}$ for rest strains. All the synthesized compounds also evaluated for antioxidant activity by DPPH method and results revealed that the compounds 8 and 16 showed antioxidant activity comparable to the positive control ascorbic acid.
\end{abstract}

Keywords: Triazoles derivatives; Anti-microbial; Anti-oxidant

\section{Introduction}

The incidence of systemic microbial infections has been increased severely due to increased of immune compromised hosts. Also, the increasing predominance of microbial resistance to huge antibiotic is becoming foremost apprehension. Consequently, the expensive treatment, toxicities and drug resistance pose new conundrum insisting constant renewed efforts in the development of new classes of antimicrobial with more specific action [1]. 1,2,4-Triazoles have attracted considerable attention in the fields of medicine and agrochemical research as well as in materials science, due to their unique structures and properties. 1,2,4-Triazole and its derivatives belong to a class of exceptionally active compounds possessing many pharmacological properties.1,2,4-Triazole and its derivatives are an important class of compounds which possess diverse agricultural, industrial and biological activities including anti-microbial Palekar et al. [2], sedative anticonvulsant Li et al. [3], anticancer Hou et al. [4], anti-inflammatory Ayse et al. [5], antitubercular Jadhav et al. [6]. In recent years, the synthesis of these heterocyclic compounds has received considerable attention. This wide range of applications has been covered by more than sixty papers in the literature, many in the form of patents.

\section{Experimental}

Starting material was obtained from commercial sources and were used without further purification. Reaction progress was observed by thin layer chromatography. Melting points were determined in open capillary automated melting point apparatus and are uncorrected. ${ }^{1} \mathrm{H}$ NMR spectra were determined by Bruker Avance II $400 \mathrm{MHz}$ NMR spectrometer using DMSO-d6 solvent and are expressed in parts per million $(\delta, \mathrm{ppm})$. NMR data are given as multiplicity (s, singlet; $\mathrm{d}$, doublet; $\mathrm{t}$, triplet; $\mathrm{m}$, multiplet) and number of protons. IR spectra were recorded on Brucker 12060280, software OPUS 7.2.139.1294 ATR spectrophotometer in a $\mathrm{KBr}$ disc. The physicochemical characteristics of synthesized compounds are presented in Table 1 . Aryl acid, ethyl esters (2a-q) and their hydrazides (3a-q). These compounds were obtained from different aryl/aroyl acids (1a-q) by the method reported in the literature [7].

\section{Synthesis of potassium dithiocarbazinate (4a-q)}

Potassium hydroxide $(0.03 \mathrm{~mol})$ was dissolved in absolute ethanol
$(50 \mathrm{~mL})$. The solution was cooled in an ice bath and acid hydrazide (3aq; $0.02 \mathrm{~mol})$ was added with stirring. To this, carbon disulfide $(0.025$ mol) was added in small portions with constant stirring. The reaction mixture was stirred continuously for $12 \mathrm{~h}$ at room temperature. The precipitated potassium dithiocarbazinate was collected by filtration, washed with anhydrous ether and dried in vacuum. The potassium salt thus obtained was used in the next step without further purification.

\section{4-amino-5-substituted-3-mercapto-(4H)-1,2,4-triazoles (5a-c)}

A suspension of potassium dithiocarbazinate derivatives (4a-q; $0.02 \mathrm{~mol})$ and hydrazine hydrate $(99 \%, 0.04 \mathrm{~mol})$ in water $(50 \mathrm{~mL})$ was refluxed for 10-15 h with occasional shaking. The color of the reaction mixture changed to light green with evolution of hydrogen sulfide gas. A homogenous mixture was obtained during the reaction process. The reaction mixture was cooled to room temperature and diluted with cold water $(20 \mathrm{~mL})$. On acidification with dil. $\mathrm{HCl}$ the required triazole was precipitated as white precipitate. It was filtered, washed with cold water, dried and recrystallized from ethanol. The compound was found pure in TLC analysis using benzene as solvent system.

Synthesis of 5,6 -dihydro-3-(substituted phenyl) $[1,2,4]$ triazolo $(3,4 b)[1,3,4]$ thiadiazine-7-one $(1-17)$

An equimolar mixture $(0.01 \mathrm{~mol})$ of 4 -amino-5-substituted-3mercapto-(4H)-1,2,4-triazoles (5a-q)and aromatic acids in chloroacetyl chloride $(10 \mathrm{~mL})$ was refluxed for $5 \mathrm{~h}$. After completion of reaction, the reaction mixture was cooled to room temperature and then poured onto crushed ice with stirring. The mixture was allowed to stand overnight and a solid mass separated out was filtered, treated with aq. ammonia and washed with cold water. It was dried and crystallized from ethanol.

*Corresponding author: Prabhakar Kumar Verma, Department of Pharmaceutical Sciences, Maharshi Dayanand University, Rohtak-124 001, Haryana, India, Tel: 9992581437; E-mail: vermapk422@rediffmail.com

Received May 04, 2017; Accepted May 16, 2017; Published May 20, 2017

Citation: Verma PK (2017) Synthesis, Antimicrobial and Antioxidant Evaluation of Novel 5, 6-dihydro-3-(substituted phenyl)[1,3,4]thiadiazine-7-one Derivatives. Med Chem (Los Angeles) 7: 880-883. doi: 10.4172/2161-0444.1000446

Copyright: @ 2017 Verma PK. This is an open-access article distributed under the terms of the Creative Commons Attribution License, which permits unrestricted use, distribution, and reproduction in any medium, provided the original author and source are credited. 


\begin{tabular}{|c|c|c|c|c|c|}
\hline Comp. & Mol.Formula & Mol.Wt. & Rf Value* $^{*}$ & M.pt. & \%Yield \\
\hline 1 & CHNOS & 248 & 0.63 & $180-182$ & 70.59 \\
\hline 2 & CHNOS & 246 & 0.56 & $172-174$ & 56.29 \\
\hline 3 & CHNOS & 322 & 0.43 & $156-158$ & 62.23 \\
\hline 4 & CHNO4S & 293 & 0.59 & $98-100$ & 65.30 \\
\hline 5 & CHCIN OS & 266 & 0.34 & $120-122$ & 68.45 \\
\hline 6 & CHNOS & 247 & 0.39 & $136-138$ & 56.10 \\
\hline 7 & CHNOS & 277 & 0.51 & $154-156$ & 54.20 \\
\hline 8 & CHCINOS & 300 & 0.24 & $143-145$ & 75.23 \\
\hline 9 & CHNOS & 322 & 0.28 & $196-198$ & 46.53 \\
\hline 10 & CHNOS & 246 & 0.34 & $103-105$ & 51.23 \\
\hline 11 & CHNOS & 232 & 0.39 & $165-167$ & 46.25 \\
\hline 12 & CHCINOS & 300 & 0.42 & $158-160$ & 64.25 \\
\hline 13 & CHNOS & 277 & 0.46 & $104-106$ & 61.35 \\
\hline 14 & CHCIN OS & 266 & 0.28 & $123-125$ & 56.36 \\
\hline 15 & CHNO & 262 & 0.37 & $174-176$ & 59.19 \\
\hline 16 & CHNOS & 246 & 0.49 & $126-128$ & 71.06 \\
\hline 17 & CHNOS & 277 & 0.51 & $135-137$ & 67.89 \\
\hline
\end{tabular}

* TLC mobile phase.* Benzene

Table 1: The Physicochemical properties of the synthesized compounds of 5,6 -dihydro-3-(substituted phenyl) $[1,2,4]$ triazolo $(3,4 b)[1,3,4]$ thiadiazine-7-one

\section{Pharmacology}

In vitro evaluation of antimicrobial activity: The antimicrobial activity was performed against Gram-positive bacteria: Staphylococcus aureus (MTCC 2901), Bacillus subtilis (MTCC 2063), and Gramnegative bacterium Escherichia coli (MTCC 1652), and several fungal strains: Candida albicans (MTCC 227), and Aspergillus niger (MTCC 8189) using tube dilution method [8]. Dilutions of test and standard compounds were prepared in double strength nutrient broth - I.P. (bacteria) or Sabouraud dextrose broth - I.P [9]. The samples were incubated at $37^{\circ} \mathrm{C}$ for $24 \mathrm{~h}$ (bacteria), at $25^{\circ} \mathrm{C}$ for $7 \mathrm{~d}($ A. Niger) and at $37^{\circ} \mathrm{C}$ for $48 \mathrm{~h}$ (C. albicans) and the results were recorded in terms of minimum inhibitory concentration (MIC). MIC was defined as the lowest conc. of compound that inhibited visible growth of microbes after incubation at $35^{\circ} \mathrm{C}$ for $24 \mathrm{~h}$. for bacteria and $48 \mathrm{~h}$ for fungi. Synthesized compounds compared to the standard drugs norfloxacin as antibacterial and fluconazole as antifungal.

In vitro evaluation of antioxidant activity: Free radical scavenging activity of synthesized compounds against stable free radical 2,2-diphenyl-2-picrylhydrazyl hydrate (DPPH), was detrmined spectrophotometrically using UV-Visible spectrophotometer. When DPPH reacts with an antioxidant compound, which can donate hydrogen, it gets reduced. Following the reduction, its deep violet colour in methanol bleached to yellow, and shows a significant absorption decrease at $517 \mathrm{~nm}$. Fifty milliliters of various concentrations $(25,50,75$ and 100$) \mu \mathrm{g} / \mathrm{ml}$ of the compounds dissolved in methanol was added to 5 $\mathrm{ml}$ of a $0.004 \%$ methanol solution of DPPH. After a $30 \mathrm{~min}$. incubation period at room temperature, the absorbance was read against a blank at $517 \mathrm{~nm}[10]$.

$$
\% I=\frac{A_{\text {control }}-A_{\text {sample }}}{A_{\text {control }}} \times 100
$$

Where,

$\mathrm{A}_{\text {control }}=$ absorbance of the control reaction

$\mathrm{A}_{\text {sample }}=$ absorbance of the test compounds

Compounds concentration providing 50\% inhibition $\left(\mathrm{IC}_{50}\right)$ was calculated from the graph plotted as inhibition percentage against compound concentration (Figure 1). Tests were carried out in triplicate and ascorbic acid was used as a positive control. Standard curve was plotted for different concentrations of ascorbic acid.

\section{Results and Discussion}

A series of 5,6-dihydro-3-(substituted phenyl) [1,2,4]triazolo(3,4-b) $[1,3,4]$ thaidiazine-one (1-17) was as outlined in Scheme 1. At first, substituted aromatic acid was reacted with ethanol in the presence of sulphuric acid and the resultant product was treated with hydrazine hydrate to yield substituted hydrazide. The later on treatment with carbon disulphide and potassium hydroxide and later again with hydrazine hydrate yielded substituted triazole and later the product on treatment chloroacetyl chloride yielded title products (1-17). The structures of synthesized compounds were characterized by IR and ${ }^{1} \mathrm{H}$ NMR. In all the cases the TLC of the product showed the single spot confirming the chromatogram for only one product. The physical constants of the title compounds are given in Table 2. Spectral details of synthesis of 5,6-dihydro-3-(substituted phenyl) $[1,2,4]$ triazolo $(3,4 b)$ $[1,3,4]$ thiadiazine-7-one.

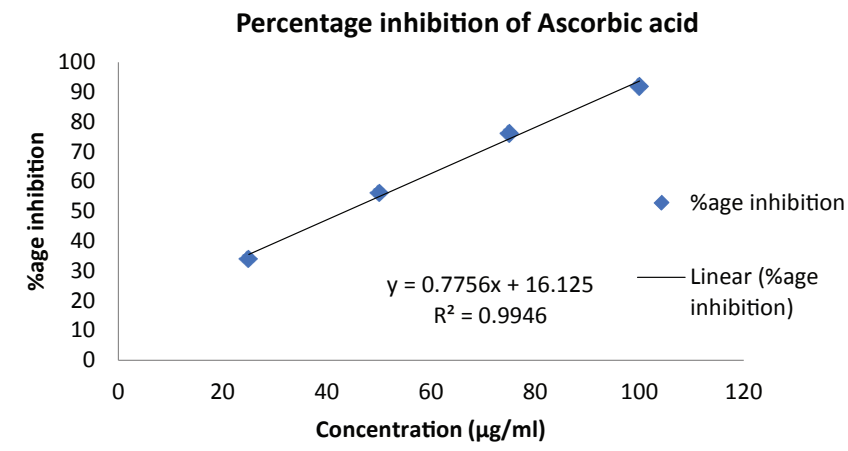

Figure 1: Result of percentage inhibition of ascorbic acid.<smiles>[R]CC(=O)NNC(S)=[SH][X]</smiles><smiles>[R]c1nnc(S)n1N</smiles>

Scheme 1: Synthesis of 5,6 -dihydro-3-(substitutedphenyl) $[1,2,4]$ triazolo $(3,4 b)[1,3,4]$ thiadiazine-7-one. 
Citation: Verma PK (2017) Synthesis, Antimicrobial and Antioxidant Evaluation of Novel 5, 6-dihydro-3-(substituted phenyl)[1,3,4]thiadiazine-7-one Derivatives. Med Chem (Los Angeles) 7: 880-883. doi: 10.4172/2161-0444.1000446

\begin{tabular}{|c|c|c|c|c|c|}
\hline \multirow{2}{*}{ Comp. } & \multicolumn{3}{|c|}{ Antibacterial } & \multicolumn{2}{c|}{ Antifungal } \\
\cline { 2 - 6 } & MIC & MIC & MIC & MIC & MIC \\
\hline & 12.50 & 06.25 & 06.25 & 12.50 & 12.50 \\
\hline & 12.50 & 06.25 & 03.12 & 06.25 & 06.25 \\
\hline & 12.50 & 12.50 & 06.25 & 12.50 & 12.50 \\
\hline & 12.50 & 12.50 & 06.25 & 12.50 & 12.50 \\
\hline & 25.00 & 12.50 & 12.50 & 12.50 & 12.50 \\
\hline & 12.50 & 12.50 & 06.25 & 12.50 & 12.50 \\
\hline & $\mathbf{0 6 . 2 5}$ & $\mathbf{0 6 . 2 5}$ & $\mathbf{0 3 . 1 2}$ & $\mathbf{0 6 . 2 5}$ & $\mathbf{1 2 . 5 0}$ \\
\hline & 12.50 & 06.25 & 06.25 & 12.50 & 12.50 \\
\hline & 12.50 & 12.50 & 06.25 & 12.50 & 12.50 \\
\hline & 06.25 & 12.50 & 06.25 & 12.50 & 12.50 \\
\hline & 25.00 & 12.50 & 12.50 & 12.50 & 12.50 \\
\hline & $\mathbf{0 6 . 2 5}$ & $\mathbf{0 6 . 2 5}$ & $\mathbf{0 3 . 1 2}$ & $\mathbf{0 6 . 2 5}$ & $\mathbf{1 2 . 5 0}$ \\
\hline & 12.50 & 12.50 & 12.50 & 12.50 & 12.50 \\
\hline & 12.50 & 06.25 & 06.25 & 06.25 & 12.50 \\
\hline & 12.50 & 12.50 & 12.50 & 12.50 & 12.50 \\
\hline & 12.50 & 06.25 & 06.25 & 12.50 & 12.50 \\
\hline Std & 25.00 & 12.50 & 12.50 & 12.50 & 25.00 \\
\hline & $\mathbf{0 1 . 5 6 *}$ & $\mathbf{0 1 . 5 6 *}$ & $\mathbf{0 1 . 5 6 *}$ & $\mathbf{0 1 . 5 6 * *}$ & $\mathbf{0 1 . 5 6 * *}$ \\
\hline
\end{tabular}

*Norfloxacin, ** Fluconazole

Table 2: Antimicrobial activity (MIC in $\mu \mathrm{g} / \mathrm{ml}$ ) of synthesized synthesis of 5,6-dihydro-3-(substituted phenyl)[1,2,4]triazolo(3,4-b)[1,3,4]thiadiazine-7-one.

\section{Compound 1 ATR $\left(\mathrm{cm}^{-1}\right)$}

IR $\mathrm{v}_{\max } \mathrm{cm}^{-1}(\mathrm{KBr}): 2987$ (C-H str., aromatic), 1707 (C=O str., carbonyl),1251 (C-O str., ester), 1660 (C=C str., aromatic ring),1567 (C-C str., aromatic in ring),3467 (O-H str.) 1063 (C-N str.),3331 (N-H str.,hydrazide). ${ }^{1} \mathrm{H}$ NMR (DMSO) $\delta ; 7.71-7.90$ (m, 4H, Ar-H), 2.187 (t,1H,NH2),1.239 (d,2H,CH2),4.14 (s, H,OH).

\section{Compound 2 ATR $\left(\mathrm{cm}^{-1}\right)$}

IR $\mathrm{v}_{\max } \mathrm{cm}^{-1}(\mathrm{KBr}): 3000$ (C-H str., aromatic), 1717 ( $\mathrm{C}=\mathrm{O}$ str., carbonyl),1251 (C-O str., ester), 1649 ( $\mathrm{C}=\mathrm{C}$ str., aromatic ring),1541 (C-C str., aromatic in ring), 1070 (C-N str.),3335(N-H str.).

\section{Compound 3 ATR $\left(\mathrm{cm}^{-1}\right)$}

IR $\mathrm{v}_{\max } \mathrm{cm}^{-1}(\mathrm{KBr}): 3034$ (C-H str., aromatic),1731 ( $\mathrm{C}=\mathrm{O}$ str., carbonyl),1257 (C-O str., ester),1681 (C=C str., aromatic ring),1571 (C-C str., aromatic in ring), 1033 (C-O str., ether), 1061(C-N str.),3407(N-H str.).

\section{Compound 4 ATR $\left(\mathrm{cm}^{-1}\right)$}

IR $\mathrm{v}_{\max } \mathrm{cm}^{-1}(\mathrm{KBr}): 3046$ (C-H str., aromatic),1730 ( $\mathrm{C}=\mathrm{O}$ str., carbonyl),1256 (C-O str., ester),1689 ( $\mathrm{C}=\mathrm{C}$ str., aromatic ring),1571 (C-C str., aromatic in ring), 1020 (C-O str., ether), 1104(C-N str.),3369(N-H str.), 3478 (OH str.).

\section{Compound 5 ATR $\left(\mathrm{cm}^{-1}\right)$}

IR $\mathrm{v}_{\max } \mathrm{cm}^{-1}(\mathrm{KBr}): 3097$ (C-H str., aromatic), 1748 ( $\mathrm{C}=\mathrm{O}$ str., carbonyl),1680 ( $\mathrm{C}=\mathrm{C}$ str., aromatic ring),1571 (C-C str., aromatic in ring),1108(C-N str.),3368(N-H str.), 745 (C-Cl str. halide).

\section{Compound 6 ATR $\left(\mathrm{cm}^{-1}\right)$}

IR $\mathrm{v}_{\max } \mathrm{cm}^{-1}(\mathrm{KBr}): 3064$ (C-H str., aromatic),1730 ( $\mathrm{C}=\mathrm{O}$ str., carbonyl), 1680 ( $\mathrm{C}=\mathrm{C}$ str., aromatic ring), 1571 (C-C str., aromatic in ring),1125(C-N str.),3368(N-H str.), 1076(C-N str.).

\section{Compound 7 ATR $\left(\mathrm{cm}^{-1}\right)$}

IR $\mathrm{v}_{\max } \mathrm{cm}^{-1}(\mathrm{KBr}): 3063$ (C-H str., aromatic),1724 ( $\mathrm{C}=\mathrm{O}$ str., carbonyl),1690 (C=C str., aromatic ring),1570 (C-C str., aromatic in ring), 1101 (C-N str.),3368 (N-H str.) 1018(C-N str.), 1506 (N-O str., nitro). ${ }^{1} \mathrm{H}$ NMR (DMSO) $\delta$; 7.414-7.7.978(m, 4H, Ar-H), 2.188 $(\mathrm{t}, 1 \mathrm{H}, \mathrm{NH}), 1.239(\mathrm{~d}, 2 \mathrm{H}, \mathrm{CH} 2)$.

\section{Compound 8 ATR $\left(\mathrm{cm}^{-1}\right)$}

IR $\mathrm{v}_{\max } \mathrm{cm}^{-1}(\mathrm{KBr}): 3087$ (C-H str., aromatic),1734 ( $\mathrm{C}=\mathrm{O}$ str., carbonyl), 1662 ( $\mathrm{C}=\mathrm{C}$ str., aromatic ring), 1567 (C-C str., aromatic in ring), 1100 (C-N str.),3365 (N-H str.,) 1007(C-N str.), 1506 (N-O str., nitro) 759 (C-Cl str.). ${ }^{1} \mathrm{H}$ NMR (DMSO) $\delta$; 7.001-7.991(m, 4H, Ar-H), $2.183(\mathrm{t}, 1 \mathrm{H}, \mathrm{NH}), 1.241(\mathrm{~d}, 2 \mathrm{H}, \mathrm{CH} 2)$.

\section{Compound 9 ATR $\left(\mathrm{cm}^{-1}\right)$}

IR $\mathrm{v}_{\max } \mathrm{cm}^{-1}(\mathrm{KBr}): 3042$ (C-H str., aromatic),1709 ( $\mathrm{C}=\mathrm{O}$ str., carbonyl),1691 ( $\mathrm{C}=\mathrm{C}$ str., aromatic ring),1549 (C-C str., aromatic in ring),1104(C-N str.),3484(N-H str.), 1062(C-N str.), 1015 (C-O str.,ether).

\section{Compound 10 ATR $\left(\mathrm{cm}^{-1}\right)$}

IR $\mathrm{v}_{\max } \mathrm{cm}^{-1}(\mathrm{KBr}): 3020$ (C-H str., aromatic),1720 ( $\mathrm{C}=\mathrm{O}$ str., carbonyl),1671 (C=C str., aromatic ring),1561 (C-C str., aromatic in ring), 1097(C-N str.),3457(N-H str.), 1013(C-N str.).

\section{Compound 11 ATR $\left(\mathrm{cm}^{-1}\right)$}

IR $\mathrm{v}_{\max } \mathrm{cm}^{-1}(\mathrm{KBr}): 3067$ (C-H str., aromatic), 1716 ( $\mathrm{C}=\mathrm{O}$ str., carbonyl),1669 (C=C str., aromatic ring), 1548 (C-C str., aromatic in ring), 1082 (C-N str.),3364 (N-H str.,) 1029(C-N str.). ${ }^{1} \mathrm{H}$ NMR (DMSO) $\delta$; 7.693-7.956(m, 6H, Ar-H), $2.180(\mathrm{t}, 1 \mathrm{H}, \mathrm{NH}), 1.237(\mathrm{~d}, 2 \mathrm{H}, \mathrm{CH} 2)$.

\section{Compound 12 ATR $\left(\mathrm{cm}^{-1}\right)$}

IR $\mathrm{v}_{\max } \mathrm{cm}^{-1}(\mathrm{KBr}): 3045$ (C-H str., aromatic),1728 ( $\mathrm{C}=\mathrm{O}$ str., carbonyl),1666 (C=C str., aromatic ring),1588 (C-C str., aromatic in ring), 1093 (C-N str.),3344 (N-H str.,), 819 (C-Cl str.,). ${ }^{1} \mathrm{H}$ NMR ppm (DMSO- d6) 7.009-7.917(m, 4H, Ar-H), 2.185 (t,1H,NH),1.245 (d,2H,CH2).

\section{Compound 13 ATR $\left(\mathrm{cm}^{-1}\right)$}

IR $\mathrm{v}_{\max } \mathrm{cm}^{-1}(\mathrm{KBr}): 3015$ (C-H str., aromatic),1730 ( $\mathrm{C}=\mathrm{O}$ str. carbonyl), 1664 ( $\mathrm{C}=\mathrm{C}$ str., aromatic ring), 1560 (C-C str., aromatic in ring), 1105 (C-N str.),3317 (N-H str.,) 1013(C-N str.), 1507 (N-O str., nitro).

\section{Compound 14 ATR $\left(\mathrm{cm}^{-1}\right)$}

IR $\mathrm{v}_{\max } \mathrm{cm}^{-1}(\mathrm{KBr}): 3049$ (C-H str., aromatic),1707 ( $\mathrm{C}=\mathrm{O}$ str., carbonyl), 1683 ( $\mathrm{C}=\mathrm{C}$ str., aromatic ring), 1561 (C-C str., aromatic in ring), 1083 (C-N str.),3370 (N-H str.,),843 (C-Cl str., halide).

\section{Compound 15 ATR $\left(\mathrm{cm}^{-1}\right)$}

IR $\mathrm{v}_{\max } \mathrm{cm}^{-1}(\mathrm{KBr}): 3063$ (C-H str., aromatic),1718 ( $\mathrm{C}=\mathrm{O}$ str., carbonyl),1674 (C=C str., aromatic ring),1561 (C-C str., aromatic in ring),1108(C-N str.),3493(N-H str.), 1063(C-N str.), 1014 (C-O

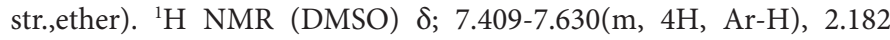
$(\mathrm{t}, 1 \mathrm{H}, \mathrm{NH}), 1.232(\mathrm{~d}, 2 \mathrm{H}, \mathrm{CH} 2)$.

\section{Compound 16 ATR $\left(\mathrm{cm}^{-1}\right)$}

IR $\mathrm{v}_{\max } \mathrm{cm}^{-1}(\mathrm{KBr}): 3097$ (C-H str., aromatic),1720 ( $\mathrm{C}=\mathrm{O}$ str., carbonyl),1679 (C=C str., aromatic ring),1574 (C-C str., aromatic in ring), 1007(C-N str.),3483(N-H str.), 993(C-N str.). ${ }^{1} \mathrm{H}$ NMR (DMSO) $\delta$; 7.130-7.958(m, 4H, Ar-H), 2.185(t,1H,NH), 1.486 (d,2H,CH2). 0.954 (d,3H,CH3). 
Citation: Verma PK (2017) Synthesis, Antimicrobial and Antioxidant Evaluation of Novel 5, 6-dihydro-3-(substituted phenyl)[1,3,4]thiadiazine-7-one Derivatives. Med Chem (Los Angeles) 7: 880-883. doi: 10.4172/2161-0444.1000446

\begin{tabular}{|l|c|c|c|c|c|}
\hline \multirow{2}{*}{ Comp } & \multicolumn{5}{|c|}{ Conc. $(\boldsymbol{\mu g} / \mathbf{m I})$} \\
\cline { 2 - 6 } & $\mathbf{2 5}$ & $\mathbf{5 0}$ & $\mathbf{7 5}$ & $\mathbf{1 0 0}$ & IC \\
\hline & 12.56 & 28.56 & 53.69 & 68.23 & 74.39 \\
\hline & 22.26 & 35.42 & 45.26 & 63.95 & 77.58 \\
\hline & 13.56 & 26.35 & 38.59 & 68.39 & 80.27 \\
\hline & 13.46 & 35.59 & 53.26 & 76.39 & 68.93 \\
\hline & 08.39 & 35.95 & 43.56 & 65.29 & 78.24 \\
\hline & 15.65 & 28.25 & 45.62 & 68.65 & 77.06 \\
\hline & 23.56 & 46.42 & 58.39 & 76.80 & 60.64 \\
\hline & $\mathbf{3 0 . 6 5}$ & $\mathbf{5 4 . 8 9}$ & $\mathbf{7 0 . 6 4}$ & $\mathbf{8 9 . 3 8}$ & $\mathbf{4 7 . 7 7}$ \\
\hline & 12.45 & 26.56 & 32.59 & 69.36 & 80.96 \\
\hline & 19.25 & 27.64 & 55.28 & 67.52 & 73.03 \\
\hline & 26.14 & 41.10 & 61.25 & 72.36 & 62.16 \\
\hline & 06.25 & 25.10 & 41.03 & 69.84 & 79.67 \\
\hline & 10.62 & 22.08 & 37.40 & 68.08 & 81.98 \\
\hline & 06.72 & 27.28 & 50.92 & 64.27 & 78.49 \\
\hline & 23.25 & 42.56 & 58.29 & 69.35 & 65.12 \\
\hline & $\mathbf{3 2 . 1 4}$ & $\mathbf{5 3 . 2 6}$ & $\mathbf{6 7 . 3 6}$ & $\mathbf{8 6 . 3 6}$ & $\mathbf{4 8 . 7 4}$ \\
\hline & 09.25 & 26.15 & 42.36 & 57.36 & 87.74 \\
\hline & $\mathbf{3 4 . 0 2}$ & $\mathbf{5 6 . 2 2}$ & $\mathbf{7 6 . 1 4}$ & $\mathbf{9 2 . 0 1}$ & $\mathbf{4 3 . 7 8}$ \\
\hline
\end{tabular}

Table 3: Percentage inhibition and IC value of the synthesized 5,6-dihydro-3 (substituted phenyl)[1,2,4]93,4-b)[1,3,4]thiadiazine-7-one derivatives.

\section{Compound 17 ATR $\left(\mathrm{cm}^{-1}\right)$}

IR $\mathrm{v}_{\max } \mathrm{cm}^{-1}(\mathrm{KBr}): 3044$ (C-H str., aromatic),1754 (C=O str., carbonyl),1674 (C=C str., aromatic ring), 1565 (C-C str., aromatic in ring), 1104 (C-N str.),3313 (N-H str.,) 1017(C-N str.), 1504 (N-O str., nitro).

\section{Antimicrobial activity}

Among the synthesized compounds most of the compounds showed good activity as compared to the Norfloxacin as antibacterial and Fluconazole as antifungal standard drugs. Compound 7 and 12 were found to be most effective against various strains used for the antimicrobial activity like $S$. aureus, B. subtilis, E. coli, Candida albicans and $A$. niger using tube dilution method having MIC $3.12 \mu \mathrm{g} / \mathrm{ml}$ for $S$. aureus and $06.25 \mu \mathrm{g} / \mathrm{ml}$ for rest strains.

\section{Antioxidant activity}

The results of antioxidant activity showed that few synthesized compounds exhibit considerable antioxidant activity. Compounds 8 and 16 showed antioxidant activity comparable to the positive control ascorbic acid (Table 3).

\section{Conclusion}

In order to develop novel antimicrobial and anticancer compounds, a series of 1,2,4-triazole derivatives was synthesized and characterized by physiochemical and spectral means. The synthesized compounds were evaluated for their antimicrobial and antioxidant potentials. Compounds 7 and 12 were found to be most effective against various strains used for the antimicrobial activity like S. aureus, B. subtilis, E. coli, Candida albicans and $A$. niger using tube dilution method having MIC $3.12 \mu \mathrm{g} / \mathrm{ml}$ for S. aureus and $06.25 \mu \mathrm{g} / \mathrm{ml}$ for rest strains. Compounds 8 and 16 showed antioxidant activity comparable to the positive control ascorbic acid.

\section{References}

1. Amir M, Javed SA, Kumar H (2008) Condensed bridgehead nitrogen heterocyclic system: Synthesis and pharmacological activities of 1,2,4-triazolo[3,4-b]-1,3,4-thiadiazole derivatives of ibuprofen and biphenyl-4-yloxy acetic acid. Eur J Med Chem 43: 2056-2066.

2. Palekar VS, Damle AJ, Shukla SR (2009) Synthesis and antibacterial activity of some novelbis-1,2,4-triazolo[3,4-b]-1,3,4- thiadiazoles and bis-4-thiazolidinone derivatives from terephthalic dihydrazide. Eur J Med Chem 44: 5112-5116.

3. Li JG, Cheng XW, Jing HJ, Li MZ, Zhe SQ (2009) Design and synthesis of 5- alkoxy-[1,2,4]triazolo[4,3-a]quinoline derivatives with anticonvulsant activity. Eur J Med Chem 44: 954-958.

4. Hou P, Sun J, Li D, Li Y, Zhang H, et al. (2011) Synthesis and antitumor activity of 1,2,4-triazoles having 1,4-benzodioxan fragment as a novel class of potent methionine aminopeptidase type II inhibitors. Bioorg Med Chem 19: 59485954.

5. Ayse UB, Banu CT, Deniz S, Ozturk El, Kazkayas I, et al. (2012) Thiazolo[3,2b]-1,2,4-triazole-5(6H)-one substituted with ibuprofen: Novel non-steroidal antiinflammatory agents with favorable gastrointestinal tolerance. Eur J Med Chem 57: 398-406.

6. Jadhav GR, Shaikh MU, Kale RP, Shiradkar MR, Gill CH (2009) SAR study of clubbed $[1,2,4]$-triazolyl with fluorobenzimidazoles as antimicrobial and antituberculosis agents. Eur J Med Chem 44: 2930-2935.

7. Hussain S, Sharma J, Amir M (2008) Synthesis and antimicrobia activities of 1,2,4-triazole and 1,3,4-thiadiazole derivatives of 5-amino-2hydroxybenzoicacid. Eur J Med Chem 5: 963-968.

8. Cappucino JG, Sherman N (1999) Microbiology - A Laboratory Manual. 4th edn. Addison Wesley, California, USA.

9. Pharmacopoeia of India (2010) Controller of Publications. Ministry of Health Department, Government of India, New Delhi, India. 1st edn. p: 37.

10. Badarinath AV, Rao KM, Chetty CM, Ramkanth S, Rajan TV, et al. (2010) A review on in-vitro antioxidant methods: comparisions, correlations and considerations. Int J Tech Res 2: 1276-1285. 